\title{
Identification of Persons by Virtue of Hand Geometry
}

\author{
Anna Plichta ${ }^{1}$, Tomasz Gaciarz ${ }^{1}$, and Szymon Szomiński ${ }^{2}$ \\ ${ }^{1}$ Cracow Univeristy of Technology Department of Computer Science, ul.Warszawska \\ 24 Cracow 31-155, Poland \\ 2 AGH University of Science and Technology, Al. A. Mickiewicza 30 Cracow 30-059, \\ Poland
}

\begin{abstract}
Biometry is a method of recognition and identification of people by means of their physical and behavioral features. These features can pertain to papillar ridges, face, hand, iris, handwriting, the way we type or even to the network of the veins within one's wrist. The biometric identification prevents the unauthorized person from accessing the ATM, personal computer, computer networks, mobile phones, household alarm systems etc.
\end{abstract}

Keywords: biometry, nearest neighbour method, image processing.

\section{Introduction}

Recently, biometric techniques have been one of the most flourishing branches of the ICT. As the amount of the processed data increases, the tightening of the access control is more and more important. The above-mentioned control pertains both to the room access and to the logical control over the individuals using the particular data or programs. The traditional techniques, based on magnetic or chip cards or on password systems no longer meet the contemporary security requirements.

The biometric methods of identification provide the high security level and are very convenient. They can be applied separately or together with the traditional access control solutions in order to prevent the room and computer networks from the nefarious use by the unauthorized people.

Currently, the most popular biometric techniques are based on:

1. papillar ridges

2. hand geometry

3. speech dynamics

4. iris pattern

Access systems based on hand geometry are often used to verify one's identity. Apart from being highly secure, they are quite cheap, easy to use. They are also thought not to breach the privacy of the users. First of all, the shape of the hand is not considered, at least by the majority of people, to be very personal or intimate feature, contrary to the fingerprints which in fact we remain 
on thousands of surfaces and things, every day. We are accustomed to giving handshakes and touching many things with our hand. Therefore, we are seldom afraid of the measuring appliance, whereas people who use iris-checking systems are often unwilling to look straight into camera lens. Secondly, features pertaining to the hand geometry do not provide the amount of information sufficient to identificate a person. Moreover, systems based on the hand geometry measurement avail of simple measurement methods and hence are inexpensive to make and easy to use. In such systems it takes much less time for the user to learn how to avail of the appliance in comparison to other biometric methods. All these features make the systems based on hand geometry measurement most widely approved biometric methods, as they seemingly do breach almost no cultural, social, psychological or religious rules.

Despite the fact that few working people lack hands, the system can be make available for, at least some, handicapped people. If necessary, the system can be integrated with other biometric techniques, especially with papillar ridges measurement or palmprints checking.

The hand biometry can function improperly if the hand is seriously wounded, break or swollen and the upper extremity injuries are perhaps the most frequent effects of accidents while working. To partially solve that problem, both hands of each worker should be put into system.

Biometric systems based on hand geometry avail of the information which pertains to the physical dimensions of the hand such as length and width of the fingers (measured in various places) or of some geometric features of the metacarpus. It should be stressed, that other features such as fingerprints, palmprints pattern, infrared photography of the hand or the pattern of blood vessels of the hand are considered separate biometric methods. They are connected to the hand, but require different processing. The majority of the above-mentioned techniques can be used in one appliance, together with the system based on the hand geometry measurment.

The data pertaining to the shape of the hand are computed from the photography of the hand taken by virtue of some particular techniques of image processing. Then, the geometric features of the hand are determined. In order to compare the hand to the pattern in the system, various classification techniques are used. The process of comparison can be considered as determining the distance between the pattern and the hands being verified. If the hand of the user matches the pattern, the distance is small (the current image of the hand and the pattern are similar enough). Otherwise, the distance is large. 12445]

\section{Recognition Process}

\subsection{Data Acquisition Methods}

The first step to identify the user is to acquire the data pertaining to the features of his hand - by means of taking photograph. It can be taken in many ways. 
For the sake of the identification method:

1. the angles between fingers should be proper

2. the photo should be taken perpendicularly to the camera lens; otherwise, the surface of the hand and fingers is different (we avail of the surfaces of fingers and hand - these are some features we want to acquire)

3. the distance between the lens should be constant; otherwise, the hand put too close to the lens would be larger and all the features would be no longer valid.

There are some professional appliances acquiring such features available to buy, for instance Handkey II or ID3D Handkey. They enable for acquiring geometric features of the hands, fingerprints or palmprints. In these appliances the span between fingers is always the same due to used pegs and the hand is isolated from the background, so the taken photograph is of very good quality.

In household, we can fix the camera and the contrastive mat on which we put hand. That method lacks pegs so the additional module correcting finger span is required.

One can also solve that problem via constructing the appliance consisting of pane of glass and the camera. The hand should be put to the glass and the lens should be installed in a fixed distance to the pane. It is typical of background in such solution to vary each time. The finger span can also vary. However, these problems can be sorted out.
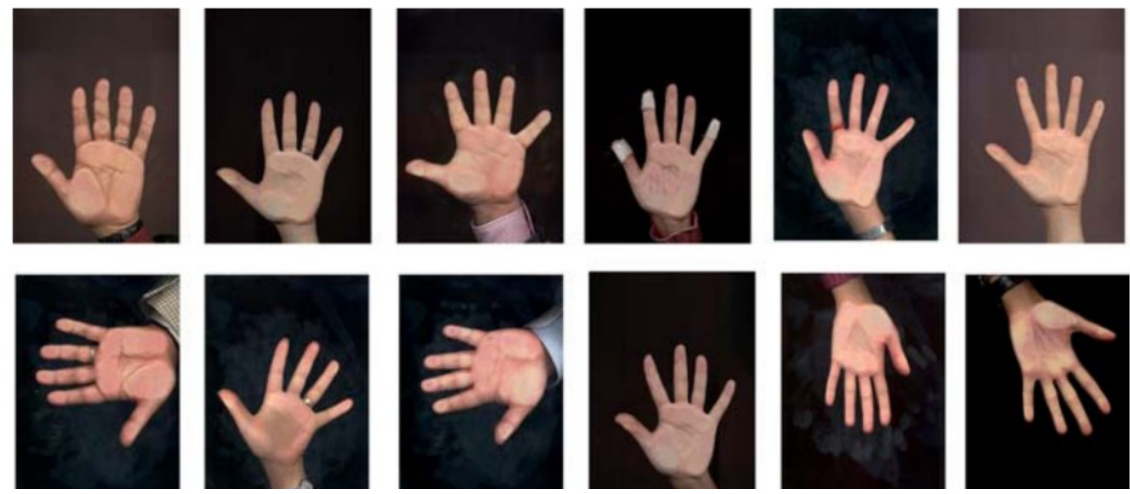

Fig. 1. Examples of the photo images of the hands on glass pane

The most user friendly method of these (and the most esthetic) is rotating the hand when the camera is fixed. For the further proceeding the system chooses the photo image being the most perpendicular to the lens. Unfortunately, the distance between the hand and the lens is problematic in this method. As we avail of many features pertaining to determining distance (fingers, hand length, circumferences, peripheries etc.), the image scaling module is required. 


\subsection{Segmentation}

In all methods which do not make use of pegs between the fingers and thus allow for variable finger span the hand segmentation module is mandatory. It is also required that for each hand the lines between the fingertops and points between them do not adjoin. There are some flaws resulting in the necessity of rejecting the particular hand images.

\subsection{Image Processing}

The acquired photo image undergoes processing. It is assumed that the image had underwent scaling and segmentation. The processing comprises the following steps:

1. background detection, binarization,

2. rotation and shifting of the hand,

3. possibly, correcting the marks left by the ring,

4. background detection, binarization,

5. correcting the angles between the fingers,

6. cutting the wrist

Table 1. Angles between the fingers and the pivot line

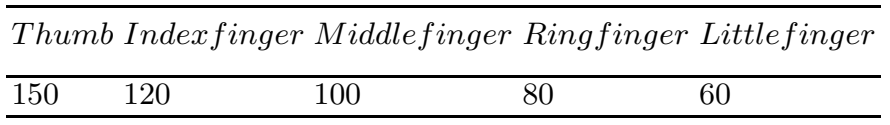

The above-mentioned figures show the steps to achieve the images of quality sufficient to be the samples in the carried out tests 4

The samples are achieved as in the Fig.2.

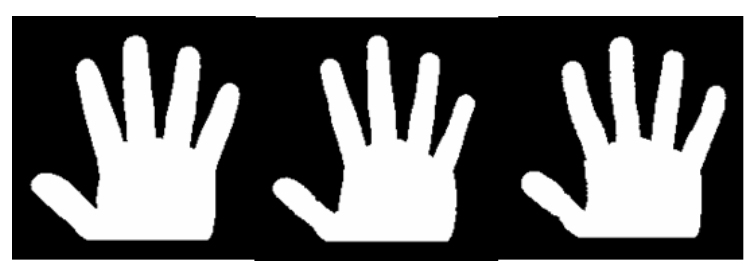

Fig. 2. The samples

The samples used in the tests were prepared and normalized by prof. dr Bulent Sankur [3] by virtue of the dedicated identification system availing of the hand geometry. The samples consisted of the photographic images taken from 755 individuals. From each individual 3 photographs were taken. The dimension of the photograph was $200 \times 200$ pixels. 


\section{The Features of the Hand Geometry}

The following set of features should be found:

1. 5x finger length

2. $5 \mathrm{x}$ base area of the finger

3. $4 \mathrm{x}$ the largest circle inscribed in the upper part of the fingers (bases of the thumb)

4. $4 \mathrm{x}$ the largest circle inscribed in the lower part of the fingers (bases of the thumb)

5. $1 \mathrm{x}$ the largest circle inscribed in thumb

6. 5x area of fingers

7. 5x circumference of the fingers

8. $4 \mathrm{x}$ distance between the lowest point of the thumb and the centers of the bases of other fingers.

9. $4 \mathrm{x}$ the sides of the rectangle around the middle of the palm (red lines in the figure)

\section{The Way of Comparison of the Features}

The input data for the application determining palm features is the vector of 39 numbers. These values are not normalized. Their scope depends on the character of the particular feature. Therefore, the features cannot be compared to one another, as each of them has completely different scope of the values. To sort that problem out, the feature vector should undergo normalization [3].

\subsection{Normalization}

The normalization process is done separately for each feature. Firstly, the minimal and maximal values of each feature are found. Then, the value of each feature is transferred into the scope $0,0-1,0$. For instance, the value of the certain feature in a sample is 15 . The minimal value is 10 and the maximal is 20 . In this case, the new value of the feature is 0,5 .

After the normalization each feature is in the scope $0,0-1,0$, so the all features have the same weight.

\subsection{The Nearest Neighbour Method}

The nearest neighbour method was chosen to comparing the samples. In consists in measuring the distance between the points represented as the feature vector in n-dimensional space and choosing the nearest points. In our case, the space has 39 dimensions.

Each individual inserted into the system is provided with some samples of his palm. That set of samples is so called class. As the points in such group lie very close to one another, we can assume that it is highly probable, that the currently examined point and the point nearest to it belong to the same class.

Below we provide the trivial example of searching for the nearest neighbour. In this case there are two classes of points 'A' and 'B'. Additionally, the space is two-dimensional. 


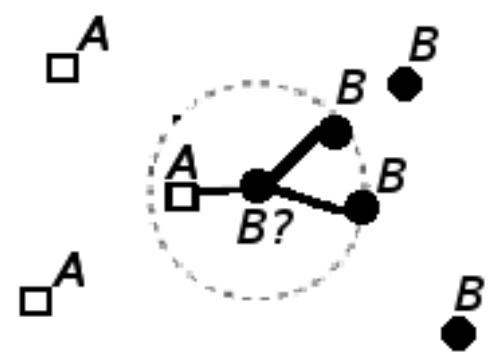

Fig. 3. Example of searching for the nearest neighbour

\subsection{Metric}

The method of measurement of the distances is also crucial. One can avail of various metrices. We have chosen the Euclidean metric. It is very popular, efficient and easy to imagine. Euclidean metric can be represented with the following formula:

$$
d_{2}(x, y)=\sqrt{\sum_{i=1}^{N}\left(x_{i}-y_{i}\right)^{2}} \quad \text { for } x, y \in \mathbb{R}^{N}
$$

\subsection{Weights}

In order to make the method more efficient we introduced the weight for each feature, too. As it is very difficult to choose the proper set of weights, it must be done automatically.

The value dispersion of each feature was considered the main factor to be taken account of while determining the weights. The more various the values were, the larger the weight was. Standard deviation is a very good indicator of dispersion and thus we took it for weights.

The standard deviation is represented with the following formula:

$$
s=\sqrt{\frac{1}{n-1} \sum_{i=1}^{n}\left(x_{i}-\bar{x}\right)^{2}}
$$

\section{Processing of the Sample and Searching for Features}

\subsection{Preparing the Contour Points Vector}

Output data - the vector embracing the points defining the contour of the palm (starting from the pixel lying one pixel-position higher than the bottom left point of the palm, clockwise). The last point of that vector is the bottom left point of the palm (Fig $4 \mathrm{~A}$ and B). 


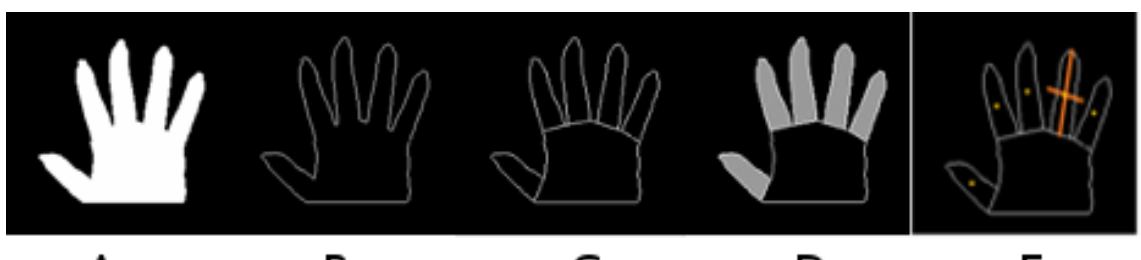
A
B
C
D
E

Fig. 4. A: Input data; B: Normalized palm image; C: Processed with the boundary-fill algorithm; D: Sum of coloured pixels; E: The middle of the line linking the middle of the nail

Creating the palm contour is a two-step process. First of all, all the white pixels are removed from the interior of the palm. Subsequently, the width of the remaining contour is one pixel and each pixel has only two neighbours. Then, the image is turned into the series of points. While searching the points the distances between these points and the referential point are also counted.

\subsection{Finding Fingertips and the Spaces between the Fingers}

The distance between the points of contour and the referential point is presented in the Fig[5] In that figure the maxima stand for the fingertips whereas the minima stand for the spaces between the fingers. According to the figure, minima and maxima can be flat. Each extremum is considered as an interval, because it cannot be unambiguously determined which pixel has the maximal value. In order to find these intervals one uses the find_extremums function.

\subsection{Finding the Features Pertaining to the Fingers}

If the key points are found, calculating the features is usually quite easy. In fact, it consists of simple mathematic operations for instance calculating the distance between the points. The function calc_fingers_feature calculates the features of the fingers.

However, the find_farrest_point function is worth mentioning because it gives back the nearest point in the given group to the point given in the second parameter. The middle of the base of the finger is chosen for that particular point.

\subsection{Finding the Points of the Base of the Palm (bottom left and bottom right point of the palm)}

The input data is the vector containing the contour points. It is intended to fill the areas of the bottom left and the bottom right point in the structure of the palm. The bottom left point of the palm is the last point of the contour. The bottom right point can be calculated assuming that it is the point of the same coordinate OY as the bottom left point and the value on the OX coordinate is as high as possible. 

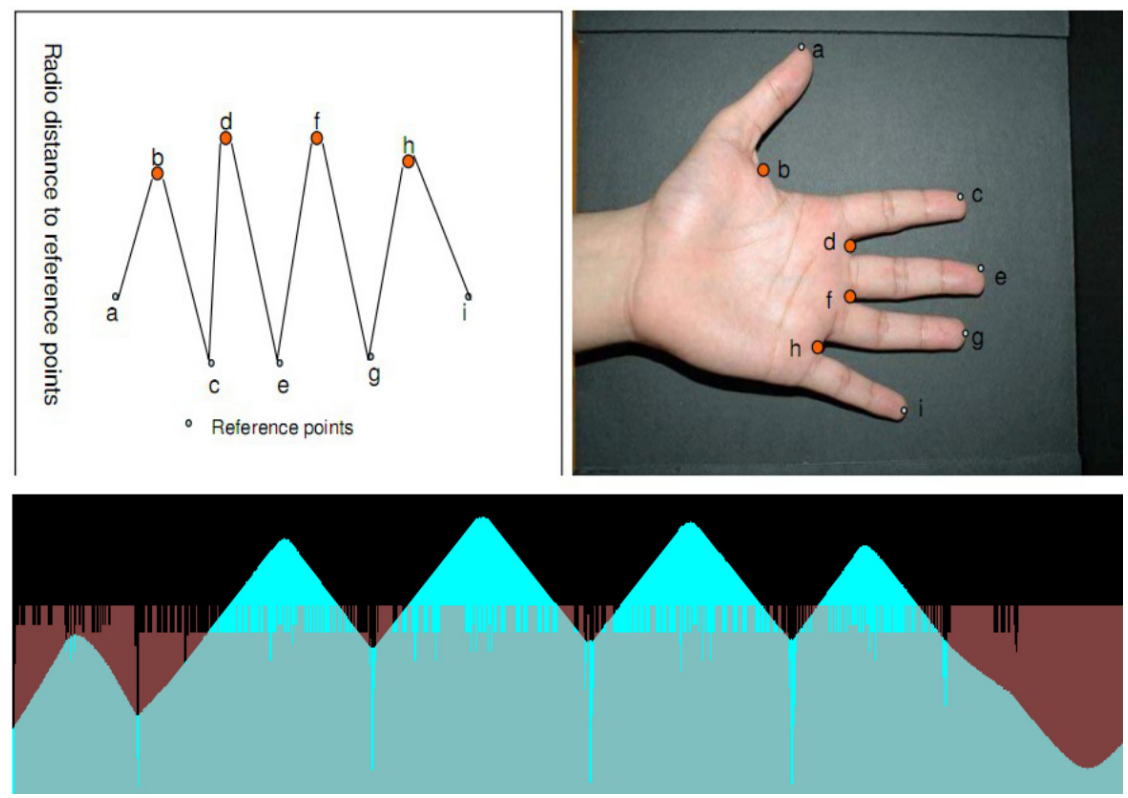

Fig. 5. The distance between the points of contour and the referential point

\subsection{Calculating the Features of the Palm}

The total area is equal to the number of the white pixels in the normalized image (Fig 4A).

The total palm legth is equal to the distance between the OY coordinate of the bottom left point of the palm and the minimal OY coordinate among the centres of the nails.

Other palm features are calculatedby means of thecalc_distance function.

\subsection{Calculatinf the Area of the Fingers}

In order to calculate the area of the fingers we avail of the algorithms pertaining to filling the boundary-areas. The input data is black image with the white palm contour, as in the Fig $4 \mathrm{~B}$.

The first step is to separate the fingers from the whole palm (by means of drawing lines linking the points between the fingers) (Fig $4 \mathrm{C}$ ).

Then, each finger is processed with the boundary-fill algorithm. All the pixels surrounded be the contour (by the closed line made of one-colour pixels) are given the same colour)

The pixels given the new colour are counted. The area of the particular finger is equal to the sum of coloured pixels in its boundary-area (Fig 4D).

The fill-seed is the pixel form which we start filling the boundary-area. It must be placed within the area to fill. In our algorithm it is the point in the middle 
of the line linking the middle of the nail and the middle of the base of the finger (Fig $4 \mathrm{E})$.

Other pixels are found thanks to the fact, that each pixel can be reached only from other pixels lying in four directions (up, down, left, right).

The above-mentioned algorithm is one of the seed fill algorithms. The fill seed is placed inside the area to fill and then it is propagated (sowed) in four directions. If the seed meets the fertile ground it fills it with colour and tries to propagate in four directions. The entire area is filled with the particular colour this way. The seed finds the fertile ground only if the pixel to propagate has different colour than the contour colour.

While sowing the number of sown seeds for the particular areas (fingers) is counted. The achieved number is equal to the area of the particular fingers.

\subsection{Finding Circles Inscribed into Fingers}

Finding circles inscribed into fingers is similar to inscribing the circle into any boundary-area for instance square, trapezoid or pentagon. To calculate the maximal circle inscribed into the boundary-area we used the Euclidean distance transform algorithm which was described in details and optimized by Pedro F. Felzenszwalb and Daniel P. Huttenlocher [6]. The principle of operation of the algorithm:

As the input data one should input the binary table (binary image);

1. the zero value stands for the boundary-area in which we want to inscribe the circle.

2. the one value stands for other pixels

As the output data we obtain the table in which all zeros were replaced with the squares of the maximal distance between the radius of the circle inscribed into the boundary-area and having the centre in a given point. Then we find the maximal value in the obtained table. In our case, it is 9. According to the algorithm, the middle of the largest circle inscribed into the given boundary-area is in the point having the biggest value (in our case, 9) The radius length is equal to the square root of the given number $(\operatorname{sqrt}(9)=3)$. [6]

\section{The Carried Out Tests}

\subsection{The Method of Carrying Out Tests}

Tests are carried out by means of the dedicated application written in c\# language. It loads the test set consisting of the consecutive feature vectors and the names of related files. [7]

Because the NN method was chosen, the application takes down nearest neighbours of the sample counting from the one nearest in relation to the Euclidean metric up to the first. Then we seek for the first element of the same class as the examined sample (it means that both samples are the representation of the 
Table 2. The exemplary excerpt from the output of the application

\begin{tabular}{llllllllll}
\hline 107: 107 & 22 & 202 & 332 & 227 & 199 & 171 & 41 & 185 & 171 \\
107: 22 & 22 & 183 & 107 & 332 & 125 & 107 & 197 & 283 & 282 \\
108: 108 & 70 & 65 & 243 & 65 & 243 & 44 & 557 & 536 & 362 \\
108: 08 & 65 & 65 & 464 & 65 & 371 & 70 & 44 & 243 & 371 \\
108: 649 & 509 & 243 & 405 & 620 & 537 & 443 & 141 & 260 & 509 \\
109: 109 & 109 & 71 & 71 & 5 & 71 & 74 & 117 & 149 & 149 \\
\hline
\end{tabular}

palm of the same user) When than element is found, we increase the value of the numerator related to the position in which it was adjusted.

The first number stands for the number of the currently examined class. The consecutive numbers stand for the classes of the nearest neighbours. As we see, in the second line the nearest neighbour for that sample has the class number 22 and the correct class was found in the fourth position.

\subsection{The Results of the Tests}

The tests were carried out of three sets of samples. The samples were chosen randomly. Each individual has 3 palm samples so the class size is 3 , too. It is a very small value and it should be increased to 5 or 10 to improve the results.

Table 3. Tests results

\begin{tabular}{lccc}
\hline \multicolumn{2}{c}{ Modification Test1(289samples) } & Test2(199samples) & Test3(2225samples) \\
\hline 1 & $90,31 \%$ & $86,93 \%$ & $65,66 \%$ \\
2 & $93,43 \%$ & $90,95 \%$ & $72,58 \%$ \\
3 & $94,46 \%$ & $92,96 \%$ & $77,08 \%$ \\
4 & $96,19 \%$ & $92,96 \%$ & $79,60 \%$ \\
5 & $96,89 \%$ & $94,47 \%$ & $81,84 \%$ \\
6 & $97,23 \%$ & $94,97 \%$ & $83,42 \%$ \\
7 & $97,92 \%$ & $96,48 \%$ & $84,54 \%$ \\
8 & $98,27 \%$ & $96,98 \%$ & $85,35 \%$ \\
9 & $98,62 \%$ & $96,98 \%$ & $86,25 \%$ \\
10 & $98,62 \%$ & $96,98 \%$ & $86,97 \%$ \\
\hline
\end{tabular}

\section{Conclusions}

According to the tests, the described method is a safe and effective identification method.

The results of the test 3 are not good. It is because the number of palm samples per each individual was very small. Increasing that number is expected to improve the results. In the test 1 the total number of samples was one less order smaller, which improved the results greatly. 
The notation of results may seem dubious. However, the notation $3-77,08 \%$ means that there is $77 \%$ probability that there is a sample of the same class as the examined one among the three nearest neighbours. That information is of some importance in relation to the systems aiding the decision whether to grant user the access to the room, content or system or not.

\section{References}

1. Yoruk, E., Dutagaci, H., Sankur, B.: Hand biometrics. Image and Vision Computing 24, 483-497 (2006)

2. Yoruk, E., Dutagaci, H., Sankur, B.: Comparative analysis of global hand apperancebased person recognition. Journal of Electronic Imaging 17(1), 011018 (2008)

3. Bulatov, Y., Jambawalikar, S., Kumar, P., Sethia, S.: Hand recognition using geometric classifiers. In: Zhang, D., Jain, A.K. (eds.) ICBA 2004. LNCS, vol. 3072, pp. 753-759. Springer, Heidelberg (2004)

4. Yoruk, E., Konukoglu, E., Sankur, B., Darbon, J.: Shape-Based Hand Recognition. IEEE Transactions on Image Processing 15(7) (July 2006)

5. Jiang, X., Xu, W.: Contactless hand recognition, http://www.cs.cmu.edu

6. Jiang, X., Xu, W., Sweeney, L., Li, Y., Gross, R., Yurovsky, D.: New directions in contact free hand recognition. In: IEEE International Conference on Image Processing, ICIP 2007, vol. 2, pp. 389-392 (2007)

7. Felzenszwalb, P.F., Huttenlocher, D.P.: Distance Transforms of Sampled Functions. Cornell Computing and Information Science (2004)

8. Bogazici University Signal and Image Processing Laboratory, http://www.busim.ee.boun.edu.tr 